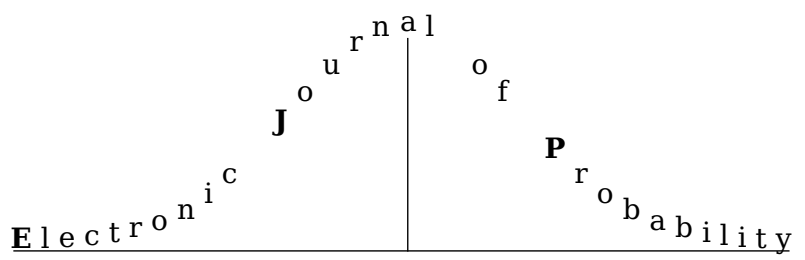

Electron. J. Probab. 26 (2021), article no. 2, 1-18.

ISSN: 1083-6489 https://doi.org/10.1214/20-EJP568

\title{
Self-similar solutions to kinetic-type evolution equations: beyond the boundary case
}

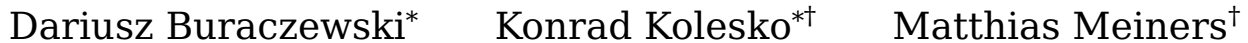

\begin{abstract}
We study the asymptotic behavior as $t \rightarrow \infty$ of a time-dependent family $\left(\mu_{t}\right)_{t \geq 0}$ of probability measures on $\mathbb{R}$ solving the kinetic-type evolution equation $\partial_{t} \mu_{t}+\mu_{t}=Q\left(\mu_{t}\right)$ where $Q$ is a smoothing transformation on $\mathbb{R}$. This problem has been investigated earlier, e.g. by Bassetti and Ladelli [Ann. Appl. Probab. 22(5): 1928-1961, 2012] and Bogus, Buraczewski and Marynych [Stochastic Process. Appl. 130(2):677-693, 2020]. Combining the refined analysis of the latter paper, which provides a probabilistic description of the solution $\mu_{t}$ as the law of a suitable random sum related to a continuous-time branching random walk at time $t$, with recent advances in the analysis of the extremal positions in the branching random walk we are able to solve the case that has been left open until now. In the course of our work, we significantly weaken the assumptions in the literature that guarantee the existence (and uniqueness) of a solution to the evolution equation $\partial_{t} \mu_{t}+\mu_{t}=Q\left(\mu_{t}\right)$.
\end{abstract}

Keywords: branching random walk; Kac model; kinetic equation; random trees; smoothing transform.

MSC2020 subject classifications: 60F05; 35B40; 60J80; 82C40.

Submitted to EJP on July 20, 2020, final version accepted on December 6, 2020.

Supersedes arXiv: 1909.00459.

\section{Introduction}

Given a sequence $A=\left(A_{1}, A_{2}, \ldots\right)$ of non-negative random variables with $N:=$ $\max \left\{j: A_{j} \neq 0\right\}<\infty$ almost surely we consider the kinetic-type evolution equation

$$
\partial_{t} \mu_{t}+\mu_{t}=Q\left(\mu_{t}\right)
$$

for a time-dependent family $\left(\mu_{t}\right)_{t \geq 0}$ of probability measures on $\mathbb{R}$ equipped with the Borel $\sigma$-algebra $\mathcal{B}(\mathbb{R})$ where (1.1) has to be understood in the weak sense and $Q$ is the

\footnotetext{
*University of Wrocław, Poland.

E-mail: dariusz. buraczewskid@uwr.edu.pl, kolesko@math.uni.wroc.pl

${ }^{\dagger}$ University of Gießen, Germany.

E-mail: konrad.kolesko@math .uni-giessen. de, matthias . meiners@math . uni-giessen.de
} 
smoothing transformation associated with $A$. More precisely, the smoothing transformation $Q$ is a self-map of $\mathcal{M}^{1}(\mathbb{R})$, the set of probability measures on $(\mathbb{R}, \mathcal{B}(\mathbb{R}))$, defined by the formula

$$
Q(\mu)=\mathcal{L}\left(\sum_{j=1}^{N} A_{j} X_{j}\right),
$$

where $\mathcal{L}(Y)$ denotes the law of a random variable $Y$ and $X_{1}, X_{2}, \ldots$ are i.i.d. and independent of $A$ with $X_{j} \sim \mu, j \in \mathbb{N}$. On the level of the Fourier transform, (1.1) corresponds to the Cauchy problem

$$
\partial_{t} \phi_{t}(\xi)+\phi_{t}(\xi)=\widehat{Q}\left(\phi_{t}\right)(\xi), \quad t \geq 0, \xi \in \mathbb{R}
$$

where $\phi_{0}$ is the Fourier transform of a given $\mu_{0} \in \mathcal{M}^{1}(\mathbb{R})$ and $\widehat{Q}$ is a self-map of the set of characteristic functions of probability measures on $(\mathbb{R}, \mathcal{B}(\mathbb{R}))$ defined by

$$
\widehat{Q}(\phi)(\xi):=\mathbb{E}\left[\prod_{j=1}^{N} \phi\left(A_{j} \xi\right)\right], \quad \xi \in \mathbb{R},
$$

for $\phi$ being the Fourier transform of some probability measure $\mu \in \mathcal{M}^{1}(\mathbb{R})$.

Under suitable assumptions (see e.g. Theorem 1.2 below or [12, Proposition 2.5]), given an initial law $\mu_{0}$, Eq. (1.1) has a unique solution, which we shall denote by $\left(\mu_{t}\right)_{t \geq 0}$ henceforth. The corresponding family of Fourier transforms will be denoted by $\left(\phi_{t}\right)_{t \geq 0}$. The behavior of the solution to (1.1) is strongly related with the spectral function $F(\theta):=\Phi(\theta) / \theta$, where

$$
\Phi(\theta):=\mathbb{E}\left[\sum_{j=1}^{N} A_{j}^{\theta}\right]-1, \quad \theta \geq 0 .
$$

\subsection{Motivation and related models in the literature}

Let us now briefly present some models that fit into the framework of Equation (1.1). Most of the models have a fixed number of $A_{j} \neq 0$, i.e., $A=\left(A_{1}, \ldots, A_{N}\right)$ with constant $N$.

The case $N=2$ and $A=(\sin U, \cos U), U$ being uniformly distributed on $[0,2 \pi)$, was considered by Kac [18] as a model for the behavior of a particle in a homogeneous gas, where particles collide at random times. It is known as the 1-dimensional Kac caricature. The distribution $\mu_{t}$ represents the law of the velocity of a randomly chosen particle and the operator $Q$ describes the change of velocity after collision of two particles. ${ }^{1}$ In subsequent works, the model was extended in various directions, for instance to non-conservative kinetic models, see e.g. [23].

The kinetic evolution equation (1.1) also found applications in models for wealth redistribution in econophysics. Loosely speaking, gas particles become agents and the velocity of a particles becomes the agent's wealth. More precisely, we consider a class of models with indistinguishable agents. The agent state is characterized by his current wealth $w \geq 0$. The interaction between two agents is described by

$$
v^{*}=p_{1} v+q_{1} w, \quad w^{*}=q_{2} v+p_{2} w,
$$

where $(v, w)$ and $\left(v^{*}, w^{*}\right)$ stand for the pre- and post-trade wealths of the two agents, respectively. The coefficients $p_{i}$ and $q_{i}$ are assumed to be random representing the risk

\footnotetext{
${ }^{1}$ Although $A_{1}, A_{2}$ are not nonnegative in the 1-dimensional Kac caricature, the model can be rephrased in the above setup as $(\sin U, \cos U)$ has the same law as $\left(\epsilon_{1}|\sin U|, \epsilon_{2}|\cos U|\right)$ with independent $\epsilon_{1}, \epsilon_{2}$ that are uniform on $\{-1,1\}$. Then one can replace $(\sin U, \cos U)$ by $(|\sin U|,|\cos U|)$ and replace the $X_{j}$ in the definition of $Q$ by $\epsilon_{j} X_{j}, j=1,2$ where $\left(\epsilon_{1}, \epsilon_{2}\right)$ and $\left(X_{1}, X_{2}\right)$ are independent, which corresponds to restricting the smoothing transform to symmetric laws on $\mathbb{R}$.
} 
of the market. The idea with random coefficients is due to the fact that agents may invest some of their money in risky assets. It is common to assume that the society's mean wealth is preserved on average, i.e., $\mathbb{E}\left[p_{1}+q_{1}+q_{2}+p_{2}\right]=2$. In our framework it can be represented by choosing

$$
A=\left(A_{1}, A_{2}\right):=\left(\epsilon p_{1}+(1-\epsilon) q_{2}, \epsilon q_{1}+(1-\epsilon) p_{2}\right),
$$

where $\epsilon$ is an independent Bernoulli variable with success parameter $\frac{1}{2}$. The conservation of mean translates to $\Phi(1)=0$. It has been shown [15, 22] that if $\Phi(r)<0$ for some $r>1$ and the expectation of $\mu_{0}$ is finite, then $\mu_{t}$ converges to some steady state $\mu_{\infty}$ which has either a Pareto tail or a slim tail. On the other hand, if $\Phi(r)>0$ for all $r>1$, then $\mu_{t} \stackrel{m}{\rightarrow} \delta_{0}$, where here and throughout the paper, $\delta_{x}$ denotes the Dirac measure with a point at $x$. In other words, a typical agent goes bankrupt. Therefore, it is natural to investigate the rate of decay of the wealth of a typical agent as $t \rightarrow \infty$.

We refer the reader to $[4,5,6,7,11]$ for examples and a more comprehensive account to the literature.

\subsection{State of the art and assumptions}

The following assumptions concerning $A$ will be relevant in the paper:

(A1) $\mathbb{P}\left(A_{1}, A_{2} \ldots \in a r^{\mathbb{Z}} \cup\{0\}\right)<1$ for all $r>1$ and $1 \leq a<r$;

(A2) There is $\vartheta>0$ such that $\Phi(\vartheta)<\infty$,

$$
\begin{aligned}
\vartheta \mathbb{E}\left[\sum_{j \geq 1} A_{j}^{\vartheta} \log A_{j}\right]+1 & =\mathbb{E}\left[\sum_{j \geq 1} A_{j}^{\vartheta}\right] \\
\text { and } \quad & \mathbb{E}\left[\sum_{j \geq 1} A_{j}^{\vartheta} \log ^{2} A_{j}\right]<\infty .
\end{aligned}
$$

(A3) For $R:=\sum_{j \geq 1} A_{j}^{\vartheta}$ and $\tilde{R}:=\sum_{j \geq 1} A_{j}^{\vartheta} \log _{+} A_{j}$ it holds that

$$
\mathbb{E}\left[R \log _{+}^{2} R\right]<\infty \text { and } \mathbb{E}\left[\tilde{R} \log _{+} \tilde{R}\right]<\infty,
$$

where $x_{ \pm}:=\max ( \pm x, 0)$ for $x \in \mathbb{R}$.

(A4) For all sufficiently small $0<\delta<1$

$$
\int_{1-\delta}^{1} \frac{\mathrm{d} s}{t-\mathbb{E}\left[t^{N}\right]}=\infty
$$

Assumption (A1) is a non-lattice assumption, while (A4) guarantees non-explosion of a related Markov branching process, see the discussion below (1.15). Notice that $\mathbb{E}[N]<\infty$ is sufficient for (A4).

If $\Phi(\theta)<\infty$, then $F(\theta)$ equals the tangent of the angle between the line segment joining $(0,0)$ and $(\theta, \Phi(\theta))$ and the positive horizontal half-axis. If $\Phi$ is defined on some open neighborhood of $\vartheta$, then the relation (1.6) states that $\Phi^{\prime}(\vartheta)=F(\vartheta)$, i.e., $\vartheta$ is the unique minimizer of $F$.

The asymptotic behavior of $\mu_{t}$ as $t \rightarrow \infty$ depends on the interplay between the minimizer $\vartheta$ and the initial distribution $\mu_{0}$. More precisely, in the existing literature usually the existence of a $\gamma \in(0,2]$ is assumed such that

$$
\phi_{0}(\xi) \sim 1-c_{ \pm}|\xi|^{\gamma} \quad \text { as } \xi \rightarrow 0^{ \pm}
$$


(i.e., $\mu_{0}$ is in the domain of normal attraction of a $\gamma$-stable law). If $\gamma>1$, it is additionally assumed that $\mu_{0}$ is centered. Then the asymptotic behavior of $\mu_{t}$ as $t \rightarrow \infty$ depends on the relation between $\vartheta$ and $\gamma$.

The vast majority of papers are treating the case where $\gamma<\vartheta$. In this case

$$
\phi_{t}\left(e^{-F(\gamma) t} \xi\right) \rightarrow \phi_{\infty}(\xi)
$$

where $\phi_{\infty}$ is the characteristic function of a non-degenerate probability distribution on $\mathbb{R}$ (cf. [11] for an analytical approach and [4] for a probabilistic interpretation).

In the recent work [12] the authors establish a connection with continuous-time branching processes which enables them to treat the boundary case $\gamma=\vartheta$ in which

$$
\phi_{t}\left(t^{\frac{1}{2 \vartheta}} e^{-F(\vartheta) t} \xi\right) \rightarrow \phi_{\infty}(\xi)
$$

again for the characteristic function $\phi_{\infty}$ of a non-degenerate probability measure on $\mathbb{R}$.

The purpose of this paper is to fill the gap in the theory of one-dimensional kinetictype equations by treating the remaining case $\vartheta<\gamma$. We actually work in even greater generality by considering $\mu_{0}$ from the class $\mathcal{M}_{\gamma}^{1}(\mathbb{R})$ for $\gamma>\vartheta$.

Definition 1.1. For $\gamma \in(0,2]$ by $\mathcal{M}_{\gamma}^{1}(\mathbb{R})$ we denote the class of probability measures with finite absolute moment of order $\gamma$, centered if $\gamma>1$.

Indeed, notice that if $0<\gamma<2$, then (1.8) implies that $\mu_{0}$ has finite absolute moments of all order $<\gamma$ [19, Theorem 11.3.2] whereas if $\gamma=2$, then $\mu_{0}$ has finite second moment [19, Theorem 11.2.1]. In both cases, if $\phi_{0}$ satisfies (1.8) with $\gamma>\vartheta$, then $\mu_{0} \in \mathcal{M}_{p}^{1}(\mathbb{R})$ for some $\vartheta<p \leq 2$. In this more general situation, we demonstrate how the asymptotic behavior of $\mu_{t}$ can be derived from recent progress on kinetic-type equations [12] and the extrema of branching random walks $[17,21]$. Our proof works under a mild $X \log X$ type moment condition (cf. assumption (A3)) and for random $N$. We mention that the assumptions about the coefficient sequence $A_{1}, A_{2}, \ldots$ in the earlier results concerning the cases $\gamma<\vartheta$ or $\gamma=\vartheta$ may be weakened analogously.

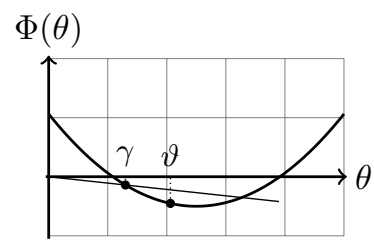

(a) The case covered by Bassetti and Ladelli [4].

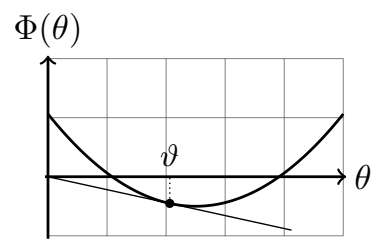

(b) The boundary case covered by Bogus et al. [12].

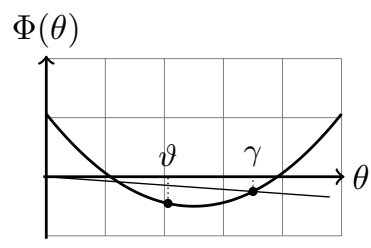

(c) The case covered by Theorem 1.3.

Figure 1: The three regimes that can occur.

The key property of the class $\mathcal{M}_{\gamma}^{1}(\mathbb{R})$ is that for a sequence $\left(X_{j}\right)_{j \in \mathbb{N}}$ of independent random variables with $\mathcal{L}\left(X_{j}\right) \in \mathcal{M}_{\gamma}^{1}(\mathbb{R})$, we have

$$
\mathbb{E}\left[\left|\sum_{j=1}^{\infty} X_{j}\right|^{\gamma}\right] \leq 2 \sum_{j=1}^{\infty} \mathbb{E}\left[\left|X_{j}\right|^{\gamma}\right], \quad n \in \mathbb{N}
$$

if the right-hand side is finite. In fact, for $\gamma \leq 1$, (1.11) holds, even with 2 replaced by 1 , by the subadditivity of $x \mapsto|x|^{\gamma}$. On the other hand, for $\gamma \in(1,2],(1.11)$ is a consequence of the von Bahr-Esseen inequality. We conclude that if $\Phi(\gamma)<\infty$, then the restriction $Q \uparrow_{\mathcal{M}_{\gamma}^{1}(\mathbb{R})}$ is a well-defined mapping from $\mathcal{M}_{\gamma}^{1}(\mathbb{R})$ to itself.

We now state the two main results of the paper. 
Theorem 1.2. Each of the following assumptions is sufficient for the existence of a solution $\left(\mu_{t}\right)_{t \geq 0}$ to the evolution equation (1.1):

(i) Assumption (A4) holds.

(ii) There exists a $\gamma \in(0,2]$ with $\mu_{0} \in \mathcal{M}_{\gamma}^{1}(\mathbb{R})$ and $\Phi(\gamma)<\infty$.

If (i) holds, the solution is unique, if (ii) holds it is the unique solution satisfying $\sup _{0 \leq s \leq t} \int|x|^{\gamma} \mu_{s}(\mathrm{~d} x)<\infty$ for all $t \geq 0$.

Theorem 1.3. Suppose that (A1) through (A3) hold with $0<\vartheta<2$. Further, assume that the initial distribution $\mu_{0}$ is not concentrated at 0 and belongs to the class $\mathcal{M}_{\gamma}^{1}(\mathbb{R})$ for some $\gamma \in(\vartheta, 2]$. Then there is a solution $\left(\mu_{t}\right)_{t \geq 0}$ to (1.1) and a probability measure $\mu_{\infty}$ on the Borel sets of $\mathbb{R}$, not concentrated in a single point, such that

$$
\lim _{t \rightarrow \infty} \phi_{t}\left(t^{\frac{3}{2 \vartheta}} e^{-F(\vartheta) t} \xi\right)=\phi_{\infty}(\xi) \quad \text { for all } \xi \in \mathbb{R},
$$

for the characteristic function $\phi_{\infty}$ of $\mu_{\infty}$.

Moreover, if $Z$ is a random variable with law $\mu_{\infty}$, then it satisfies the following stochastic fixed-point equation

$$
Z \stackrel{\text { law }}{=} U^{F(\vartheta)} \sum_{j=1}^{N} A_{j} Z^{(j)},
$$

where $Z^{(1)}, Z^{(2)}, \ldots$ are independent copies of $Z, U$ is uniformly distributed on $(0,1)$ and $U, A=\left(A_{1}, A_{2}, \ldots\right)$ and $\left(Z^{(j)}\right)_{j \in \mathbb{N}}$ are independent.

Equation (1.12) is called fixed-point equation of the smoothing transformation. A lot of information on $Z$ can be extracted from the fact that (the law of) $Z$ satisfies (1.12). More precisely, we are in the situation with nonnegative weights $U^{F(\vartheta)} A_{j}, j \in \mathbb{N}$ and possibly real-valued $Z$. In this setup, the equation has been solved in [2]. An important parameter for (1.12) is the characteristic index $\alpha>0$, the minimal positive solution of the equation $m(t)=1$ where

$$
m(t)=\mathbb{E}\left[\sum_{j \geq 1} U^{t F(\vartheta)} A_{j}^{t}\right]=\frac{\Phi(t)+1}{\frac{t}{\vartheta} \Phi(\vartheta)+1} .
$$

Notice that $m(\vartheta)=1$. If $\Phi(t)=\infty$ for all $t<\vartheta$, then $\alpha=\vartheta$. If $\Phi(t)<\infty$ for some $0 \leq t<\vartheta$, then also $m(t)<\infty$ and, as a consequence, $m$ is finite on $[t, \vartheta]$. Then $m$ is differentiable (from the left) at $t=\vartheta$. The derivative equals 0 at $\vartheta$ if (1.6) holds. By the convexity of $m$, we again infer that $\alpha=\vartheta$. If $m(t)<\infty$ for some $t<\vartheta$, Theorems 2.1 and 2.2 of [2] imply that

$$
Z \stackrel{\text { law }}{=} W^{1 / \vartheta} Y_{\vartheta}
$$

where $W \geq 0$ is the limit of an associated derivative martingale (namely, that of the branching random walk with first generation positions given by $-\log \left(U^{\Phi(\vartheta)} A_{j}^{\vartheta}\right), j \in \mathbb{N}$ with $A_{j}>0$ ) and $Y_{\vartheta}$ is a strictly $\vartheta$-stable random variable independent of $W$. From this representation, one can deduce various properties of the distribution of $Z$. For instance, one may deduce the tail-behavior of $Z$ from that of $W$ and $Y_{\vartheta}$ using the main result of the recent paper [13]. We now briefly explain why the random variable $W$ given by Theorems 2.1 and 2.2 of [2] can be chosen as the limit of the derivative martingale as stated above. The cited theorems state that $W$ is a solution of the stochastic fixed-point equation

$$
W \stackrel{\mathrm{law}}{=} \sum_{j=1}^{N} U^{\Phi(\vartheta)} A_{j}^{\vartheta} W^{(j)}
$$


where $W^{(1)}, W^{(2)}, \ldots$ are i.i.d. copies of $W$ and independent of $U, A_{1}, A_{2}, \ldots$ By [8, Theorem 3], the law of $W$ is uniquely determined by this fixed-point equation (up to a positive scale factor). On the other hand, under the present assumptions, the derivative martingale of a branching random walk with first generation positions given by $-\log \left(U^{\Phi(\vartheta)} A_{j}^{\vartheta}\right), j=1, \ldots, N$ converges almost surely to some nonnegative, nondegenerate limit by [1, Proposition A.3(iii)]. The limit of the derivative martingale is also a solution to (1.14), see [8].

Let us demonstrate how Theorem 1.3 translates to the particular economical model described above by the random vector $\left(A_{1}, A_{2}\right)$ defined in (1.5). In this model, $\Phi(1)=0$ and if $\Phi(r)=0$ for some $r<1$, then the typical agent goes bankrupt (provided the initial wealth distribution $\mu_{0}$ has finite mean). In this case, the minimizer $\vartheta$ is in the interval $(r, 1)$ and $F(\vartheta)<0$. If the tail of the initial distribution is heavy enough (i.e., if $\gamma<\vartheta$ ), then the wealth of a typical agent behaves like $e^{F(\gamma) t}$, which diverges for $\gamma<r$. Next, in the boundary case $\gamma=\vartheta$, the correct asymptotic is $t^{-\frac{1}{2 \vartheta}} e^{F(\vartheta) t}$. Our result deals with the remaining case where the tail of the initial distribution is not heavy enough, i.e., $\gamma>\vartheta$. In particular, this covers the situation where the first moment exists, which seems to be the most natural case in this context. In this case, the wealth of a typical agent decays like $t^{-\frac{3}{2 \vartheta}} e^{F(\vartheta) t}$ as $t \rightarrow \infty$.

\subsection{Representation of solutions: the branching random walk connection}

Given an initial distribution $\mu_{0}$ and the random vector $A$, we give a representation of $\mu_{t}$ as the law of a continuous-time branching random walk at time $t$. The exact form of representation was developed in [12], see also [4] and the references therein for earlier results.

We write $\mathcal{I}:=\bigcup_{n \in \mathbb{N}_{0}} \mathbb{N}^{n}$ where $\mathbb{N}^{0}=\{\varnothing\}$ contains only the empty tuple $\varnothing$. For $u \in \mathcal{I}, u=\left(u_{1}, \ldots, u_{m}\right)$, we also write $u_{1} \ldots u_{m}$ and if $v=\left(v_{1}, \ldots, v_{n}\right)$, we write $u v$ for $\left(u_{1}, \ldots, u_{m}, v_{1}, \ldots, v_{n}\right)$. Further, if $k \leq m$, we set $\left.u\right|_{k}:=u_{1} \ldots u_{k}$. Finally, for $u \in \mathcal{I}$, we use the notation $|u|=n$ for $u \in \mathbb{N}^{n}$.

Throughout the paper, we work on a fixed probability space $(\Omega, \mathcal{F}, \mathbb{P})$ on which two independent families $(A(u), E(u))_{u \in \mathcal{I}}$ and $\left(X_{u}\right)_{u \in \mathcal{I}}$ of random vectors and random variables, respectively, are defined such that

- the $(A(u), E(u)), u \in \mathcal{I}$ are independent and identically distributed (i.i.d.) copies of $(A, E)$ where $A$ is given and $E$ is an independent unit-mean exponential random variable;

- the $X_{u}, u \in \mathcal{I}$ are i.i.d. copies of a random variable $X$ with $\mathcal{L}(X)=\mu_{0}$.

For convenience, we denote quantities related to the ancestor without the label $\varnothing$, i.e., $(A, E)=(A(\varnothing), E(\varnothing))$ etc.

We now recursively define a continuous-time Markov branching process $\left(\mathcal{Y}_{t}\right)_{t \geq 0}$ starting with one particle, the ancestor, denoted by $\varnothing$, at time $t=0$. The birth-time of the ancestor is $\sigma(\varnothing)=0$. If a particle labelled $u \in \mathcal{I}$ is born at time $\sigma(u)$, it lives an exponential lifetime $E(u)$ until $\sigma(u)+E(u)$ at which time it dies and simultaneously gives birth to new particles labelled $u 1, u 2, \ldots$. For a particle $u=u_{1} \ldots u_{m} \in \mathcal{I}$, we write

$$
S(u):=-\sum_{k=1}^{m} \log A_{u_{k}}\left(\left.u\right|_{k-1}\right)
$$

for its position on the real line. The position $S(u)=\infty$, we consider as a ghost type: the corresponding individual is never born. We write

$$
\mathcal{I}_{t}:=\{u \in \mathcal{I}: S(u)<\infty \text { and } \sigma(u) \leq t<\sigma(u)+E(u)\}
$$


for the set of labels pertaining to individuals alive at time $t$. Finally, we write

$$
\mathcal{Z}_{t}:=\sum_{u \in \mathcal{I}_{t}} \delta_{S(u)}
$$

for the continuous-time branching random walk at time $t \geq 0$. Throughout the paper, we denote by $\left(T_{n}\right)_{n \in \mathbb{N}_{0}}$ the sequence of points in increasing order of a homogeneous Poisson process with intensity 1 and a point at the origin, i.e., $T_{0}=0$. We suppose that $\left(T_{n}\right)_{n \in \mathbb{N}_{0}}$ is independent of the $(A(u), E(u)), X_{u}, u \in \mathcal{I}$. The Laplace transform at $\theta \geq 0$ of the intensity measure of $\mathcal{Z}_{t}$ is given by

$$
\begin{aligned}
m(t, \theta) & :=\mathbb{E}\left[\sum_{u \in \mathcal{I}_{t}} e^{-\theta S(u)}\right]=\mathbb{E}\left[\sum_{n \geq 0} \sum_{|u|=n} e^{-\theta S(u)} \mathbb{1}_{\{\sigma(u) \leq t, \sigma(u)+E(u)>t\}}\right] \\
& =\sum_{n \geq 0} \mathbb{E}\left[\sum_{|u|=n} e^{-\theta S(u)}\right] \mathbb{P}\left(T_{n} \leq t<T_{n+1}\right) \\
& =\sum_{n \geq 0}(\Phi(\theta)+1)^{n} e^{-t} \frac{t^{n}}{n !}=e^{t \Phi(\theta)} .
\end{aligned}
$$

By classical results ([16, Theorem 9.1, p. 107] with an analytic proof or [24, Theorem 5] for a probabilistic proof) it follows that the set $\mathcal{I}_{t}$ is finite almost surely for all $t \geq 0$, provided (A4) holds. In particular, the sum

$$
U_{t}:=\sum_{u \in \mathcal{I}_{t}} e^{-S(u)} X_{u}
$$

is a well-defined, finite random variable. On the other hand, if $\mathcal{L}(X) \in \mathcal{M}_{\theta}^{1}(\mathbb{R})$ and $\Phi(\theta)<\infty$ for some $0<\theta \leq 2$, then the right hand side of (1.16) converges in $L^{\theta}$ by (1.11) and (1.15).

The connection between the continuous-time branching random walk $\left(\mathcal{Z}_{t}\right)_{t \geq 0}$ and the kinetic-type evolution equation (1.1) is established in the following proposition, which implies Theorem 1.2.

Proposition 1.4. In the situation of Theorem 1.2, each of the conditions (i) and (ii) of the theorem implies the existence of a solution $\left(\mu_{t}\right)_{t \geq 0}$ to (1.1) given by

$$
\mu_{t}=\mathcal{L}\left(U_{t}\right), \quad t \geq 0 .
$$

If (i) holds, the solution is unique. If (ii) holds, then it is the unique solution satisfying $\sup _{0 \leq s \leq t} \int|x|^{\gamma} \mu_{s}(\mathrm{~d} x)<\infty$ for all $t \geq 0$.

Proof. First, we provide an equation, which is equivalent to (1.2) and easier to work with. If $\phi_{t}$ is a solution to the kinetic-type equation (1.2), then it satisfies the integral equation

$$
e^{t} \phi_{t}(\xi)-\phi_{0}(\xi)=\int_{0}^{t} e^{s} \widehat{Q}\left(\phi_{s}\right)(\xi) \mathrm{d} s, \quad t \geq 0, \xi \in \mathbb{R},
$$

and vice versa. Recall that $E=E(\varnothing)$ is a unit-mean exponential random variable independent of all $S(u)$ and $X_{u}, u \in \mathcal{I}$. With the convention that $\phi_{t}=\phi_{0}$ for $t \leq 0$, in view of (1.3), the above equation can be rewritten as

$$
\phi_{t}(\xi)=e^{-t} \phi_{0}(\xi)+\int_{0}^{t} e^{-s} \widehat{Q}\left(\phi_{t-s}\right)(\xi) \mathrm{d} s=\mathbb{E}\left[\prod_{u \in \mathcal{I}_{t}^{1}} \phi_{t-E}\left(e^{-S(u)} \xi\right)\right],
$$


valid for $t \geq 0$ and $\xi \in \mathbb{R}$ where

$$
\mathcal{I}_{t}^{n}=\mathcal{I}_{t}^{n, 1} \cup \mathcal{I}_{t}^{n, 2}:=\left\{u \in \mathcal{I}_{t}:|u| \leq n\right\} \cup\{u \in \mathcal{I}: \sigma(u) \leq t,|u|=n\}
$$

for any $n \in \mathbb{N}_{0}$. We show that the function $\psi_{t}(\xi):=\mathbb{E}\left[\exp \left(\mathrm{i} \xi U_{t}\right)\right]$ satisfies (1.18) provided that condition (i) or (ii) of Theorem 1.2 holds. Indeed, for any $t \geq 0$,

$$
\mathbb{E}\left[\exp \left(\mathrm{i} \xi U_{t}\right) \mid(A, E)\right]=\mathbb{1}_{\{E>t\}} \mathbb{E}[\exp (\mathrm{i} \xi X)]+\mathbb{1}_{\{E \leq t\}} \prod_{|u|=1} \psi_{t-E}\left(e^{-S(u)} \xi\right),
$$

and therefore ${ }^{2}$

$$
\psi_{t}(\xi)=\mathbb{E}\left[\mathbb{1}_{\{E>t\}} \phi_{0}(\xi)+\mathbb{1}_{\{E \leq t\}} \prod_{|u|=1} \psi_{t-E}\left(e^{-S(u)} \xi\right)\right]=\mathbb{E}\left[\prod_{u \in \mathcal{I}_{t}^{1}} \psi_{t-E}\left(e^{-S(u)} \xi\right)\right]
$$

Let us also note that if $\mu_{0} \in \mathcal{M}_{\gamma}^{1}(\mathbb{R})$ and $\Phi(\gamma)<\infty$, then by (1.11) and (1.15) we infer

$$
\mathbb{E}\left[\left|U_{t}\right|^{\gamma}\right] \leq 2 e^{t \Phi(\gamma)} \int|x|^{\gamma} \mu_{0}(\mathrm{~d} x)
$$

which is locally bounded. Moreover, $\mathbb{E}\left[U_{t}\right]=0$ for all $t \geq 0$ if $\gamma>1$.

Now we prove that $\psi_{t}$ is the only solution to (1.18). Let $\left(\phi_{t}\right)_{t \geq 0}$ be any solution with initial condition $\phi_{0}$. Inductively, for any $n \in \mathbb{N}_{0}$, iterating (1.18) we get

$$
\begin{aligned}
\phi_{t}(\xi) & =\mathbb{E}\left[\prod_{u \in \mathcal{I}_{t}^{n}} \phi_{t-\sigma(u)}\left(e^{-S(u)} \xi\right)\right] \\
& =\mathbb{E}\left[\prod_{u \in \mathcal{I}_{t}^{n, 1}} \phi_{t-\sigma(u)}\left(e^{-S(u)} \xi\right) \cdot \prod_{u \in \mathcal{I}_{t}^{n} \backslash \mathcal{I}_{t}^{n, 1}} \phi_{t-\sigma(u)}\left(e^{-S(u)} \xi\right)\right] .
\end{aligned}
$$

We show that, for fixed $t \geq 0$,

$$
\prod_{u \in \mathcal{I}_{t}^{n} \backslash \mathcal{I}_{t}^{n, 1}} \phi_{t-\sigma(u)}\left(e^{-S(u)} \xi\right) \rightarrow 1 \quad \text { in } L^{1} \text { as } n \rightarrow \infty
$$

This is clear if (i) holds since then, with probability one, $\mathcal{I}_{t}$ is finite and hence the product above is eventually indexed by the empty set. On the other hand, if (ii) and the additional assumption $\sup _{0 \leq s \leq t} \int|x|^{\gamma} \mu_{s}(\mathrm{~d} x)<\infty$ for all $t \geq 0$ hold, using [14, Theorem 1 on p. 295] we infer existence of a function $t \mapsto C(t) \geq 0$ such that

$$
\sup _{0 \leq s \leq t}\left|1-\phi_{s}(\xi)\right| \leq C(t)|\xi|^{\gamma} \text { for all } \xi \in \mathbb{R} \text {. }
$$

Using this together with $\mathcal{I}_{t}^{n} \backslash \mathcal{I}_{t}^{n, 1}=\{u \in \mathcal{I}:|u|=n, S(u)+E(u) \leq t\}$ and the elementary inequality $\left|1-\prod_{k} z_{k}\right| \leq \sum_{k}\left|1-z_{k}\right|$, valid for $z_{k} \in \mathbb{C}$ with $\left|z_{k}\right| \leq 1$, we conclude

$$
\left|1-\prod_{u \in \mathcal{I}_{t}^{n} \backslash \mathcal{I}_{t}^{n, 1}} \phi_{t-\sigma(u)}\left(e^{-S(u)} \xi\right)\right| \leq C(t)|\xi|^{\gamma} \sum_{\substack{|u|=n, \sigma(u)+E(u) \leq t}} e^{-\gamma S(u)} .
$$

Since

$$
\mathbb{E}\left[\sum_{\substack{|u|=n, \sigma(u)+E(u) \leq t}} e^{-\gamma S(u)}\right] \leq(\Phi(\gamma)+1)^{n} \sum_{k>n} e^{-t} \frac{t^{k}}{k !} \rightarrow 0,
$$

\footnotetext{
${ }^{2}$ Notice that the a.s. finiteness of $U_{t}$ and $N<\infty$ a.s. are the only assumptions required to draw this conclusion.
} 
as $n$ goes to infinity, we conclude (1.19). Consequently, in both cases we have

$$
\begin{aligned}
\phi_{t}(\xi) & =\lim _{n \rightarrow \infty} \mathbb{E}\left[\prod_{u \in \mathcal{I}_{t}^{n}} \phi_{t-\sigma(u)}\left(e^{-S(u)} \xi\right)\right]=\lim _{n \rightarrow \infty} \mathbb{E}\left[\prod_{u \in \mathcal{I}_{t}^{n, 1}} \phi_{0}\left(e^{-S(u)} \xi\right)\right] \\
& =\lim _{n \rightarrow \infty} \mathbb{E}\left[\exp \left(\mathrm{i} \xi \sum_{u \in \mathcal{I}_{t}^{n, 1}} e^{-S(u)} X_{u}\right)\right]=\mathbb{E}\left[\exp \left(\mathrm{i} \xi U_{t}\right)\right]=\psi_{t}(\xi) .
\end{aligned}
$$

The above result provides an explicit form of the solution to Equation (1.1). Therefore, in order to prove our main result we need to find an appropriate scaling of the random sum (1.16) leading to a nontrivial limit law as $t \rightarrow \infty$. For this purpose, first applying the Croft-Kingman lemma [20], we reduce the problem of describing convergence along any sequence to convergence along arbitrary lattice sequences (Section 2). Finally, we show the existence of the limit along lattice sequences (Section 3).

\section{Reduction to the lattice case}

The goal of this section is to prove the following lemma.

Lemma 2.1. Suppose that (A1) through (A3) holds, $\mu_{0} \in \mathcal{M}_{\gamma}^{1}(\mathbb{R})$ for some $\gamma \in(\vartheta, 2]$ and that, for any fixed $\delta>0$,

$$
(n \delta)^{\frac{3}{2 \vartheta}} e^{-F(\vartheta) n \delta} U_{n \delta} \stackrel{\text { d }}{\rightarrow} Z_{\delta} \quad \text { as } n \rightarrow \infty
$$

for some non-degenerate random variable $Z_{\delta}$. Then

$$
t^{\frac{3}{2 \vartheta}} e^{-F(\vartheta) t} U_{t} \stackrel{\text { d }}{\rightarrow} Z_{1} \quad \text { as } t \rightarrow \infty .
$$

Moreover, the random variable $Z:=Z_{1}$ satisfies (1.12).

The lemma above is proved in several steps. First, for $p>0$ and $x \geq 0$, we define $f_{p}(x):=\left(1+\log _{+}^{p} x\right) x$ and notice that $f_{p}$ is superadditive, i.e., $f_{p}(x+y) \geq f_{p}(x)+f_{p}(y)$ for all $x, y \geq 0$. It is also subadditive up to a multiplicative constant, namely,

$$
f_{p}(x+y) \leq 2\left(1+\log ^{p} 2\right)\left(f_{p}(x)+f_{p}(y)\right) \text { for all } x, y \geq 0 .
$$

Similarly, $f_{p}$ is submultiplicative in the sense that

$$
f_{p}(x y) \leq 2^{p} f_{p}(x) f_{p}(y) \text { for all } x, y \geq 0 .
$$

Further, let

$$
h_{p}(x):=\int_{0}^{x}\left(\frac{p}{e}\right)^{p} t \mathbb{1}_{\left[0, e^{p}\right]}(t)+(\log t)^{p} \mathbb{1}_{\left(e^{p}, \infty\right)}(t) \mathrm{d} t .
$$

Then $h_{p}$ is convex with concave derivative $h_{p}^{\prime}$. Further, $f_{p}$ and $h_{p}$ are asymptotically equivalent, i.e.,

$$
\lim _{x \rightarrow \infty} \frac{f_{p}(x)}{h_{p}(x)}=1 .
$$

Consequently, since $h_{p}^{\prime}(0)=0<1=f_{p}^{\prime}(0)$, there is some $C_{p}>0$ such that

$$
h_{p}(x) \leq C_{p} f_{p}(x) \text { for all } x \geq 0 .
$$

We start with a technical lemma. 
Lemma 2.2. Suppose that

$$
\mathbb{E}\left[f_{p}\left(\sum_{|u|=1} e^{-S(u)}\right)\right]<\infty
$$

for some $p>0$. Then there is a constant $C>0$ such that, for any $t \geq 0$,

$$
\begin{aligned}
\mathbb{E}\left[\sum_{u \in \mathcal{I}} f_{p}\left(e^{-S(u)}\right) \mathbb{1}_{\{\sigma(u) \leq t\}}\right] & \leq C e^{C t} \\
\text { and } \mathbb{E}\left[f_{p}\left(\sum_{u \in \mathcal{I}_{t}} e^{-S(u)}\right)\right] & \leq C e^{C t} .
\end{aligned}
$$

Proof. Throughout the proof, if some quantity depending on $t \geq 0$ is bounded by $C e^{C t}$ for all $t \geq 0$ and some constant $C>0$, then we say that the quantity grows at most exponentially fast. Using induction on $n$, the superadditivity of $f_{p}$ and (2.4), we infer

$$
\mathbb{E}\left[\sum_{|u|=n} f_{p}\left(e^{-S(u)}\right)\right] \leq q^{n},
$$

for some $q>1$. Then

$$
\begin{aligned}
\mathbb{E}\left[\sum_{u \in \mathcal{I}} f_{p}\left(e^{-S(u)}\right) \mathbb{1}_{\{\sigma(u) \leq t\}}\right] & =\sum_{n \geq 0} \mathbb{E}\left[\sum_{|u|=n} f_{p}\left(e^{-S(u)}\right) \mathbb{1}_{\{\sigma(u) \leq t\}}\right] \\
& \leq \sum_{n \geq 0} q^{n} \mathbb{P}\left(T_{n} \leq t\right)=\frac{q e^{t(q-1)}-1}{q-1}<\infty,
\end{aligned}
$$

proving (2.8). Turning to the proof of (2.9), we first notice that, for every $t \in \mathbb{R}$,

$$
\sum_{u \in \mathcal{I}_{t}} e^{-S(u)} \leq \sum_{u \in \mathcal{I}} e^{-S(u)} \mathbb{1}_{\{\sigma(u) \leq t\}}=: M^{t} .
$$

(Here, for $t<0$, both sums are empty and hence have value 0 .) Since $f_{p}$ is monotone, it suffices to prove that $\mathbb{E}\left[f_{p}\left(M^{t}\right)\right]$ grows at most exponentially fast. Since $f_{p}(x) \geq x$ for any $x \geq 0$, we conclude from (2.8) that $H(t):=\mathbb{E}\left[M^{t}\right] \leq C^{\prime} e^{C^{\prime} t}$ for all $t \geq 0$ and an appropriate constant $C^{\prime}>0$. Thus, by (2.3), for all $t \geq 0$,

$$
\begin{aligned}
\mathbb{E}\left[f_{p}\left(M^{t}\right)\right] & =\mathbb{E}\left[f_{p}\left(M^{t}-H(t)+H(t)\right)\right] \\
& \leq 2\left(1+\log ^{p} 2\right)\left(\mathbb{E}\left[f_{p}\left(\left|M^{t}-H(t)\right|\right)\right]+f_{p}(H(t))\right) \\
& \leq 2\left(1+\log ^{p} 2\right)\left(\mathbb{E}\left[f_{p}\left(\left|M^{t}-H(t)\right|\right)\right]+f_{p}\left(C^{\prime} e^{C^{\prime} t}\right)\right) .
\end{aligned}
$$

Therefore, it suffices to prove that $\mathbb{E}\left[f_{p}\left(\left|M^{t}-H(t)\right|\right)\right]$ grows at most exponentially fast in $t$. By (2.6), there is a constant $C^{\prime \prime}$ such that

$$
\mathbb{E}\left[f_{p}\left(\left|M^{t}-H(t)\right|\right)\right] \leq 2 \mathbb{E}\left[h_{p}\left(\left|M^{t}-H(t)\right|\right)\right]+C^{\prime \prime} \text { for all } t \geq 0,
$$

so it suffices to prove that $\mathbb{E}\left[h_{p}\left(\left|M^{t}-H(t)\right|\right)\right]$ grows at most exponentially fast in $t$. To this end, let $\varnothing=u_{1}, u_{2}, \ldots \in \mathcal{I}$ be a deterministic enumeration of $\mathcal{I}$ such that, with $\mathcal{I}^{(n)}=\left\{u_{1}, \ldots, u_{n}\right\}$, the sequence $\left(\mathcal{I}^{(n)}\right)_{n \in \mathbb{N}}$ is a strictly increasing sequence of subtrees of $\mathcal{I}$, and let

$$
\mathcal{F}_{\mathcal{I}^{(n)}}:=\sigma\left((A(u), E(u)): u \in \mathcal{I}^{(n)}\right) .
$$

Then $M_{n}^{t}:=\mathbb{E}\left[M^{t} \mid \mathcal{F}_{\mathcal{I}^{(n)}}\right], n \in \mathbb{N}$ is a (uniformly integrable) martingale. By the martingale convergence theorem, $M_{n}^{t} \rightarrow M^{t}$ a.s. and in $L^{1}$. Consider the martingale differences

$$
M_{n}^{t}-M_{n-1}^{t}=\sum_{u \in \mathcal{I}}\left(\mathbb{E}\left[e^{-S(u)} \mathbb{1}_{\{\sigma(u) \leq t\}} \mid \mathcal{F}_{I^{(n)}}\right]-\mathbb{E}\left[e^{-S(u)} \mathbb{1}_{\{\sigma(u) \leq t\}} \mid \mathcal{F}_{I^{(n-1)}}\right]\right), n \in \mathbb{N}
$$


If $u$ is not a strict descendant of $u_{n}$, i.e., if there is no $v \in \mathcal{I}$ with $|v| \geq 1$ such that $u=u_{n} v$, then $\left(A\left(u_{n}\right), E\left(u_{n}\right)\right)$ is independent of the $\sigma$-algebra generated by $e^{-S(u)} \mathbb{1}_{\{\sigma(u) \leq t\}}$ and $\mathcal{F}_{I^{(n-1)}}$, hence, $\mathbb{E}\left[e^{-S(u)} \mid \mathcal{F}_{I^{(n)}}\right]=\mathbb{E}\left[e^{-S(u)} \mid \mathcal{F}_{I^{(n-1)}}\right]$ a. s. Hence, with

$$
\begin{aligned}
D_{n}^{t} & :=\mathbb{E}\left[\sum_{|v| \geq 1} e^{-S\left(u_{n} v\right)} \mathbb{1}_{\left\{\sigma\left(u_{n} v\right) \leq t\right\}} \mid \mathcal{F}_{I^{(n)}}\right] \\
& =\sum_{j=1}^{N\left(u_{n}\right)} e^{-S\left(u_{n} j\right)} \mathbb{E}\left[\sum_{v \in \mathcal{I}} e^{-\left(S\left(u_{n} j v\right)-S\left(u_{n} j\right)\right)} \mathbb{1}_{\left\{\sigma\left(u_{n} j v\right)-\sigma\left(u_{n} j\right) \leq t-\sigma\left(u_{n} j\right)\right\}} \mid \mathcal{F}_{\mathcal{I}^{(n-1)}}\right] \\
& =\sum_{j=1}^{N\left(u_{n}\right)} e^{-S\left(u_{n} j\right)} H\left(t-\sigma\left(u_{n} j\right)\right) \quad \text { a.s., }
\end{aligned}
$$

we have

$$
M_{n}^{t}-M_{n-1}^{t}=D_{n}^{t}-\mathbb{E}\left[D_{n}^{t} \mid \mathcal{F}_{\mathcal{I}^{(n-1)}}\right] \text { a.s. }
$$

Since $h_{p}(0)=0, h_{p}$ is increasing and convex with concave derivative, we may apply the Topchiī-Vatutin inequality [3] and infer

$$
\begin{aligned}
\mathbb{E}\left[h_{p}\left(\left|M^{t}-H(t)\right|\right)\right] & \leq 2 \sum_{n=1}^{\infty} \mathbb{E}\left[h_{p}\left(\left|M_{n}^{t}-M_{n-1}^{t}\right|\right)\right] \\
& \leq 2 \sum_{n=1}^{\infty}\left(\mathbb{E}\left[h_{p}\left(D_{n}^{t}\right)\right]+\mathbb{E}\left[h_{p}\left(\mathbb{E}\left[D_{n}^{t} \mid \mathcal{F}_{\mathcal{I}^{(n-1)}}\right]\right)\right]\right) \\
& \leq 4 \sum_{n=1}^{\infty} \mathbb{E}\left[h_{p}\left(D_{n}^{t}\right)\right] \leq 4 C_{p} \sum_{n=1}^{\infty} \mathbb{E}\left[f_{p}\left(D_{n}^{t}\right)\right],
\end{aligned}
$$

where we have applied Jensen's inequality for conditional expectations in the next-to-last step and (2.7) in the last step. Here, using the definition of $D_{n}^{t}, H(t) \leq C^{\prime} e^{C^{\prime} t} \mathbb{1}_{[0, \infty)}(t)$ for all $t \in \mathbb{R}$, and (2.4) (twice), we find

$$
\begin{aligned}
\sum_{n=1}^{\infty} \mathbb{E}\left[f_{p}\left(D_{n}^{t}\right)\right] & =\sum_{u \in \mathcal{I}} \mathbb{E}\left[f_{p}\left(\sum_{j=1}^{N(u)} e^{-S(u j)} H(t-\sigma(u j))\right)\right] \\
& \leq \sum_{u \in \mathcal{I}} \mathbb{E}\left[f_{p}\left(\sum_{j=1}^{N(u)} e^{-S(u j)} \mathbb{1}_{\{\sigma(u j) \leq t\}} C^{\prime} e^{C^{\prime} t}\right)\right] \\
& \leq 2^{p} f_{p}\left(C^{\prime} e^{C^{\prime} t}\right) \sum_{u \in \mathcal{I}} \mathbb{E}\left[f_{p}\left(\sum_{j=1}^{N(u)} e^{-S(u j)} \mathbb{1}_{\{\sigma(u j) \leq t\}}\right)\right] \\
& \leq 4^{p} f_{p}\left(C^{\prime} e^{C^{\prime} t}\right) \sum_{u \in \mathcal{I}} \mathbb{E}\left[f_{p}\left(e^{-S(u)}\right) \mathbb{1}_{\{\sigma(u) \leq t\}} f_{p}\left(\sum_{j=1}^{N(u)} e^{-(S(u j)-S(u))}\right)\right] \\
& =4^{p} f_{p}\left(C^{\prime} e^{C^{\prime} t}\right) \mathbb{E}\left[\sum_{u \in \mathcal{I}} f_{p}\left(e^{-S(u)}\right) \mathbb{1}_{\{\sigma(u) \leq t\}}\right] \mathbb{E}\left[f_{p}\left(\sum_{|u|=1} e^{-S(u)}\right)\right]
\end{aligned}
$$

which is finite and grows at most exponentially fast by (2.11) and since the last expectation is finite by assumption.

Lemma 2.3. Suppose that the assumptions of Lemma 2.1 hold. Then the family $\left(U_{t}\right)_{t \geq 0}$ is continuous in $L^{\vartheta}$, i.e., $\mathbb{E}\left[\left|U_{t}-U_{s}\right|^{\vartheta}\right] \rightarrow 0$ as $s \rightarrow t$. In particular, if $a:[0, \infty) \rightarrow[0, \infty)$ is a deterministic nonnegative continuous function, then also $\left(a(t) U_{t}\right)_{t \geq 0}$ is continuous in $L^{\vartheta}$. 
Proof. Define $g(t, s):=\mathbb{E}\left[\left|U_{t}-U_{s}\right|^{\vartheta}\right]$ for $s, t \geq 0$. We first show that $g(t, 0) \rightarrow 0$ as $t \rightarrow 0$. Notice that $U_{0}=X_{\varnothing}=X \sim \mu_{0}$. Then, with $S_{t}:=\{E \leq t\}$ denoting the event that there was a split in the interval $[0, t]$, we have $\mathbb{P}\left(S_{t}\right)=1-e^{-t}$. Consequently,

$$
g(t, 0)=\mathbb{E}\left[\left|U_{t}-X\right|^{\vartheta} \mathbb{1}_{S_{t}}\right] \leq 2^{\vartheta} \mathbb{E}\left[\left|U_{t}\right|^{\vartheta} \mathbb{1}_{S_{t}}\right]+2^{\vartheta} \mathbb{E}\left[|X|^{\vartheta}\right]\left(1-e^{-t}\right) .
$$

The second summand on the right-hand side vanishes as $t \rightarrow 0$, so it remains to consider the first one. For the function $h_{1}$ defined by (2.5) with $p=1$ the expectation $\mathbb{E}\left[h_{1}\left(\left|U_{t}\right|^{\vartheta}\right)\right]$ remains bounded as $t$ goes to 0 . We postpone the proof of this fact and first show how it implies $\mathbb{E}\left[\left|U_{t}\right|^{\vartheta} \mathbb{1}_{S_{t}}\right] \rightarrow 0$ as $t \rightarrow 0$. Indeed, since $h_{1}$ is convex and grows superlinearly fast, the Legendre-Fenchel transform $h_{1}^{*}(y):=\sup _{x \geq 0}\left(x y-h_{1}(x)\right)$ of the function $h_{1}$ is finite on the half-line $[0, \infty)$ and $h_{1}^{*}(y) \rightarrow \infty$ as $y \rightarrow \infty$. From the definition of $h_{1}^{*}$, we conclude that $x y \leq h_{1}(x)+h_{1}^{*}(y)$ for all $x, y \geq 0$ (a generalized version of Young's inequality) and $h_{1}^{*}(y)>0$ iff $y>0$. Using these inequalities, (2.7) and (2.4), we infer, for any $s_{t}>1$,

$$
\begin{aligned}
\mathbb{E}\left[\left|U_{t}\right|^{\vartheta} \mathbb{1}_{S_{t}}\right] & \leq \mathbb{E}\left[h_{1}\left(s_{t}^{-1}\left|U_{t}\right|^{\vartheta}\right)\right]+\mathbb{E}\left[h_{1}^{*}\left(s_{t} \mathbb{1}_{S_{t}}\right)\right] \\
& \leq s_{t}^{-1} C \mathbb{E}\left[f_{1}\left(\left|U_{t}\right|^{\vartheta}\right)\right]+h_{1}^{*}\left(s_{t}\right) \mathbb{P}\left(S_{t}\right)
\end{aligned}
$$

where $C>0$ is an appropriate constant. Taking now $s_{t} \rightarrow \infty$ such that $h_{1}^{*}\left(s_{t}\right)=$ $\mathbb{P}\left(S_{t}\right)^{-1 / 2}=\left(1-e^{-t}\right)^{-1 / 2} \rightarrow 0$ as $t \rightarrow 0$, we conclude that the second summand tends to 0 as $t \rightarrow \infty$. Regarding the first, notice that (2.6) together with $\lim \sup _{t \rightarrow 0} \mathbb{E}\left[h_{1}\left(\left|U_{t}\right|^{\vartheta}\right)\right]<\infty$ implies that it also tends to 0 as $t \rightarrow 0$.

We now turn to the proof of the fact that $\limsup _{t \rightarrow 0} \mathbb{E}\left[h_{1}\left(\left|U_{t}\right|^{\vartheta}\right)\right]<\infty$. For technical reasons, we need to replace $h_{1}\left(|x|^{\vartheta}\right)$ by a function of the same order of growth with more convenient properties. To this end, first suppose that $\vartheta \in(1,2)$ and consider $g_{\vartheta}^{\prime \prime}:[0, \infty) \rightarrow[0, \infty)$

$$
g_{\vartheta}^{\prime \prime}(u):= \begin{cases}\frac{1}{e(2-\vartheta)}, & \text { for } u \leq e^{1 /(2-\vartheta)}, \\ u^{\vartheta-2} \log u, & \text { for } u \geq e^{1 /(2-\vartheta)} .\end{cases}
$$

The function $g_{\vartheta}^{\prime \prime}$ is nonnegative, continuous and non-increasing, hence $g_{\vartheta}:[0, \infty) \rightarrow[0, \infty)$, defined by

$$
g_{\vartheta}(x)=\int_{0}^{x} \int_{0}^{t} g_{\vartheta}^{\prime \prime}(u) \mathrm{d} u \mathrm{~d} t, \quad x \geq 0,
$$

is convex with concave derivative. Two applications of the direct half of Karamata's theorem [10, Proposition 1.5.8] imply that

$$
g_{\vartheta}(x) \sim \frac{x^{\vartheta} \log x}{\vartheta(\vartheta-1)} \text { as } x \rightarrow \infty .
$$

Since $h_{1}\left(x^{\vartheta}\right) \sim \vartheta x^{\vartheta} \log x$ as $x \rightarrow \infty$, we have $\limsup _{t \rightarrow 0} \mathbb{E}\left[h_{1}\left(\left|U_{t}\right|^{\vartheta}\right)\right]<\infty$ if and only if $\limsup _{t \rightarrow 0} \mathbb{E}\left[g_{\vartheta}\left(\left|U_{t}\right|\right)\right]<\infty$ Further, since we also have $f_{1}\left(x^{\vartheta}\right) \sim x^{\vartheta}$ as $x \rightarrow 0$ and $f_{1}\left(x^{\vartheta}\right) \sim \vartheta x^{\vartheta} \log x$ as $x \rightarrow \infty$, whereas $g_{\vartheta}(x) \sim x^{2} /(2 e(2-\vartheta))$ as $x \rightarrow 0$, there exists a constant $C_{\vartheta}>0$ such that $g_{\vartheta}(x) \leq C_{\vartheta} f_{1}\left(x^{\vartheta}\right)$ for all $x \geq 0$. A combination of this inequality, the (conditional) Topchii-Vatutin inequality (recall that $\mathbb{E}[X]=0$ in this case) and (2.4) yields

$$
\begin{aligned}
\mathbb{E}\left[g_{\vartheta}\left(\left|U_{t}\right|\right)\right] & \leq 2 \mathbb{E}\left[\sum_{u \in \mathcal{I}_{t}} g_{\vartheta}\left(e^{-S(u)}\left|X_{u}\right|\right)\right] \leq 2 C_{\vartheta} \mathbb{E}\left[\sum_{u \in \mathcal{I}_{t}} f_{1}\left(e^{-\vartheta S(u)}\left|X_{u}\right|^{\vartheta}\right)\right] \\
& \leq 4 C_{\vartheta} \mathbb{E}\left[\sum_{u \in \mathcal{I}_{t}} f_{1}\left(e^{-\vartheta S(u)}\right) f_{1}\left(\left|X_{u}\right|^{\vartheta}\right)\right]
\end{aligned}
$$


which is bounded by Lemma 2.2 and $\mathbb{E}\left[|X|^{\gamma}\right]<\infty$. If $\vartheta=1$, the situation is easier and the above argument works with $g_{\vartheta}(x):=h_{1}(x), x \geq 0$ as this function is convex with concave derivative. If $\vartheta<1$, then we define $g_{\vartheta}^{\prime}:[0, \infty) \rightarrow[0, \infty)$ via

$$
g_{\vartheta}^{\prime}(t):= \begin{cases}\frac{t^{\vartheta-1}}{1-\vartheta}, & \text { for } t \leq e^{1 /(1-\vartheta)}, \\ t^{\vartheta-1} \log t, & \text { for } t \geq e^{1 /(1-\vartheta)}\end{cases}
$$

and $g_{\vartheta}(x):=\int_{0}^{x} g_{\vartheta}^{\prime}(t) \mathrm{d} t, x \geq 0$. Again by Karamata's theorem, $g_{\vartheta}(x) \sim \frac{1}{\vartheta} x^{\vartheta} \log x$ as $x \rightarrow \infty$, i.e., $g_{\vartheta}(x)$ is of the same order of growth as $f_{1}\left(x^{\vartheta}\right)$ as $x \rightarrow \infty$. Similarly, $g_{\vartheta}(x)=\frac{1}{\vartheta} x^{\vartheta}$ and $f_{1}\left(x^{\vartheta}\right)=x^{\vartheta}$ for small $x$. Consequently, again we find a constant $C_{\vartheta}>0$ such that $g_{\vartheta}(x) \leq f_{1}\left(x^{\vartheta}\right)$ for all $x \geq 0$. On the other hand, as $g_{\vartheta}^{\prime}$ is non-increasing, $g_{\vartheta}$ is subadditive and hence

$$
\mathbb{E}\left[g_{\vartheta}\left(\left|U_{t}\right|\right)\right] \leq \mathbb{E}\left[\sum_{u \in \mathcal{I}_{t}} g_{\vartheta}\left(e^{-S(u)}\left|X_{u}\right|\right)\right] \leq 2 C_{\vartheta} \mathbb{E}\left[\sum_{u \in \mathcal{I}_{t}} f_{1}\left(e^{-\vartheta S(u)}\right) f_{1}\left(\left|X_{u}\right|^{\vartheta}\right)\right] .
$$

Again by Lemma 2.2, this is bounded for sufficiently small $t$.

Now let $s, t \geq 0$. By conditioning with respect to $\mathcal{F}_{t \wedge s}$, the $\sigma$-algebra containing all information up to and including time $t \wedge s$, and using the Markov property, we infer

$$
\begin{aligned}
g(t, s) & =\mathbb{E}\left[\left|U_{t}-U_{s}\right|^{\vartheta}\right] \leq \mathbb{E}\left[\sum_{u \in \mathcal{I}_{t \wedge s}} e^{-\vartheta S(u)} g(|t-s|, 0)\right] \\
& =m(t \wedge s, \vartheta) \cdot g(|t-s|, 0) \rightarrow 0
\end{aligned}
$$

as $t$ is kept fixed and $s \rightarrow t$ by the first part of the proof.

Proof of Lemma 2.1. Suppose that (2.1) holds for all fixed $\delta>0$. Let $f: \mathbb{R} \rightarrow \mathbb{R}$ be differentiable with derivative $f^{\prime}$ such that both $f$ and $f^{\prime}$ are continuous and bounded. Define, for $t \geq 0$,

$$
h(t):=\mathbb{E}\left[f\left(t^{\frac{3}{2 \vartheta}} e^{-F(\vartheta) t} U_{t}\right)\right] .
$$

By (2.1), we have

$$
h(n \delta) \rightarrow \mathbb{E}\left[f\left(Z_{\delta}\right)\right] \text { as } n \rightarrow \infty,
$$

for all $\delta>0$. If we can show that $h$ is continuous, then the Croft-Kingman lemma [20, Theorem 2] applies and gives that $\mathbb{E}\left[f\left(Z_{\delta}\right)\right]$ is independent of $\delta$ and that $\lim _{t \rightarrow \infty} h(t)=$ $\mathbb{E}\left[f\left(Z_{1}\right)\right]$. Since the bounded continuously differentiable functions with bounded derivative are convergence determining on $\mathbb{R}$, this implies (2.2).

We now turn to the proof of the continuity of $h$. For any $x, y \in \mathbb{R}$, we have

$$
|f(x)-f(y)| \leq\left(\left\|f^{\prime}\right\|_{\infty} \cdot|x-y|\right) \wedge\left(2\|f\|_{\infty}\right) \leq C|x-y|^{\vartheta \wedge 1}
$$

for some finite constant $C \geq 0$. Consequently, for any $s, t \geq 0$,

$$
|h(s)-h(t)| \leq C \mathbb{E}\left[\left|s^{\frac{3}{2 \vartheta}} e^{-F(\vartheta) s} U_{s}-t^{\frac{3}{2 \vartheta}} e^{-F(\vartheta) t} U_{t}\right|^{\vartheta \wedge 1}\right] .
$$

The latter expression tends to 0 as $s \rightarrow t$ by Lemma 2.3 .

To prove the second part of the Lemma note that the process $U_{t}$ satisfies the following branching relation

$$
U_{t+s} \stackrel{\text { law }}{=} \sum_{u \in \mathcal{I}_{t}} e^{-S(u)} U_{s, u}
$$

where $\left(U_{s, u}\right)_{u}$ are independent copies of $U_{s}$, independent of the process up to time $t$. Then (2.13) entails

$$
U_{t} \stackrel{\text { law }}{=} \mathbb{1}_{\{E>t\}} X+\mathbb{1}_{\{E \leq t\}} \sum_{k=1}^{N} A_{k} U_{t-E, k}
$$


and therefore

$$
t^{\frac{3}{2 \vartheta}} e^{-F(\vartheta) t} U_{t} \stackrel{\text { law }}{=} \mathbb{1}_{\{E>t\}} t^{\frac{3}{2 \vartheta}} e^{-F(\vartheta) t} X+\mathbb{1}_{\{E \leq t\}} e^{-F(\vartheta) E} \sum_{k=1}^{N} t^{\frac{3}{2 \vartheta}} e^{-F(\vartheta)(t-E)} A_{k} U_{t-E, k} .
$$

Now passing with $t$ to $\infty$ we conclude that $Z_{1}$ satisfies (1.12).

\section{Convergence along lattices}

Throughout the whole Section 3, we fix some $\delta>0$ and prove that (2.1) holds for a non-degenerate random variable $Z_{\delta}$.

\subsection{Properties of the skeleton branching random walk}

The sequence of point processes $\left(\mathcal{Z}_{n \delta}\right)_{n \in \mathbb{N}_{0}}$ forms a discrete-time (or skeleton) branching random walk, in which each individual produces offspring with displacement relative to its position given by the points of an independent copy of the point process $\mathcal{Z}_{\delta}$. In this section, we shall discuss the properties of this branching random walk that are relevant to us.

As $\delta$ is kept fixed throughout Section 3, we abbreviate $m(\delta, \theta)$, defined in (1.15), by $m(\theta)$. For $n \in \mathbb{N}_{0}$ and $u \in \mathcal{I}_{n \delta}$, we define

$$
V(u):=\vartheta S(u)+n \log m(\vartheta)=\vartheta S(u)+n \delta \Phi(\vartheta) .
$$

By the definition of $\vartheta$, we have

$$
\mathbb{E}\left[\sum_{u \in \mathcal{I}_{\delta}} e^{-V(u)}\right]=1 \quad \text { and } \quad \mathbb{E}\left[\sum_{u \in \mathcal{I}_{\delta}} V(u) e^{-V(u)}\right]=0,
$$

i.e., the branching random walk $\left(\sum_{u \in \mathcal{I}_{n \delta}} \delta_{V(u)}\right)_{n \in \mathbb{N}_{0}}$ is in the boundary case. ${ }^{3}$ Indeed, the first equation in (3.2) follows from (1.15). Regarding the second, first notice that, by (1.6) and (1.4)

$$
\mathbb{E}\left[\sum_{|u|=1} \vartheta S(u) e^{-\vartheta S(u)}\right]=-\vartheta \mathbb{E}\left[\sum_{j \geq 1} A_{j}^{\vartheta} \log A_{j}\right]=-\mathbb{E}\left[\sum_{j \geq 1} A_{j}^{\vartheta}\right]+1=-\Phi(\vartheta)
$$

and hence, by the many-to-one lemma (see e.g. [8, Proposition 11]), for every $n \in \mathbb{N}_{0}$,

$$
\mathbb{E}\left[\sum_{|u|=n} \vartheta S(u) e^{-\vartheta S(u)}\right]=-n \Phi(\vartheta)(\Phi(\vartheta)+1)^{n-1} .
$$

Consequently,

$$
\begin{gathered}
\mathbb{E}\left[\sum_{u \in \mathcal{I}_{\delta}} V(u) e^{-V(u)}\right]=\mathbb{E}\left[\sum_{u \in \mathcal{I}_{\delta}} \vartheta S(u) e^{-\vartheta S(u)}\right] e^{-\delta \Phi(\vartheta)}+\delta \Phi(\vartheta) \mathbb{E}\left[\sum_{u \in \mathcal{I}_{\delta}} e^{-V(u)}\right] \\
=e^{-\delta \Phi(\vartheta)} \sum_{n \geq 0} \mathbb{E}\left[\sum_{|u|=n} \vartheta S(u) e^{-\vartheta S(u)} \mathbb{1}_{\{\sigma(u) \leq \delta<\sigma(u)+E(u)\}}\right]+\delta \Phi(\vartheta) \\
=-\Phi(\vartheta) e^{-\delta \Phi(\vartheta)} \sum_{n \geq 1} n(\Phi(\vartheta)+1)^{n-1} \mathbb{P}\left(T_{n} \leq \delta<T_{n+1}\right)+\delta \Phi(\vartheta)=0
\end{gathered}
$$

since $\mathbb{P}\left(T_{n} \leq \delta<T_{n+1}\right)=e^{-\delta} \frac{\delta^{n}}{n !}$ for all $n \in \mathbb{N}_{0}$. Moreover, the skeleton branching random walk $\left(\mathcal{Z}_{n \delta}\right)_{n \in \mathbb{N}_{0}}$ is non-lattice by (A1) and satisfies

$$
\mathbb{E}\left[\sum_{u \in \mathcal{I}_{\delta}} V(u)^{2} e^{-V(u)}\right] \in(0, \infty)
$$

\footnotetext{
${ }^{3}$ This notion was coined by Biggins and Kyprianou in [8].
} 
The latter follows from Lemma 3.6 in [12]. As a corollary of Lemma 2.2, we get the following:

Proposition 3.1. Suppose that assumptions (A2) and (A3) are fulfilled. Then for the branching random walk defined by (3.1) it holds

$$
\begin{aligned}
& \mathbb{E}\left[\left(\sum_{u \in \mathcal{I}_{\delta}} e^{-V(u)}\right) \log _{+}^{2}\left(\sum_{u \in \mathcal{I}_{\delta}} e^{-V(u)}\right)\right]<\infty \text { and } \\
& \mathbb{E}\left[\left(\sum_{u \in \mathcal{I}_{\delta}} e^{-V(u)} V(u)_{+}\right) \log _{+}\left(\sum_{u \in \mathcal{I}_{\delta}} e^{-V(u)} V(u)_{+}\right)\right]<\infty .
\end{aligned}
$$

Proof. An application of Lemma 2.2 with $V=\vartheta S, p=2$ and $t=\delta$ gives (3.4). For (3.5) note that

$$
\vartheta S(u)_{+} e^{-\vartheta S(u)} \leq e^{-\tilde{S}(u)},
$$

where $\tilde{S}(u):=\sum_{i=1}^{|u|} \vartheta\left(S\left(u_{i}\right)-S\left(u_{i-1}\right)\right)-\log \left(1+\vartheta\left(S\left(u_{i}\right)-S\left(u_{i-1}\right)\right)_{+}\right)$. Hence the application of Lemma 2.2 with $V=\tilde{S}, p=1$ and $t=\delta$ finishes the proof.

We have now checked that the assumptions of [21, Theorem 1.1] hold and infer, with $V_{n}(u):=V(u)-\frac{3}{2} \log (n)$ for $u \in \mathcal{I}_{n \delta}$,

$$
\mathcal{Z}_{n}^{\circ}:=\sum_{u \in \mathcal{I}_{n \delta}} \delta_{V_{n}(u)} \stackrel{\mathrm{d}}{\rightarrow} \mathcal{Z}_{\infty}^{\circ}
$$

where $\mathcal{Z}_{\infty}^{\circ}$ is a point process on $\mathbb{R}$ satisfying $\mathcal{Z}_{\infty}^{\circ}((-\infty, 0])<\infty$ a. s. Here, the convergence in distribution is in the space of locally finite point measures equipped with the topology of vague convergence. For $k \in \mathbb{N}$, define $P_{k}:=\inf \left\{t \in \mathbb{R}: \mathcal{Z}_{\infty}^{\circ}((-\infty, t]) \geq k\right\}$, that is, $-\infty<P_{1} \leq P_{2} \leq P_{3} \leq \ldots$ and $\mathcal{Z}_{\infty}^{\circ}=\sum_{k \in \mathbb{N}} \delta_{P_{k}}$. Then [17, Formula (5.4)] gives

$$
\sum_{k \in \mathbb{N}} e^{-\beta P_{k}}<\infty \quad \mathbb{P} \text {-a.s. }
$$

for every $\beta>1$. For simplicity of notation, suppose that $\mathcal{Z}_{\infty}^{\circ}$ is defined on the probability space $(\Omega, \mathcal{F}, \mathbb{P})$ and that it is independent of the families $(A(u), E(u))_{u \in \mathcal{I}}$ and $\left(X_{u}\right)_{u \in \mathcal{I}}$. In particular, $\left(X_{k}\right)_{k \in \mathbb{N}}$ is independent of $\mathcal{Z}_{\infty}^{\circ}$. We consider the following random sums

$$
Z_{n}^{*}:=\sum_{k=1}^{n} e^{-\frac{P_{k}}{v}} X_{k}, \quad n \in \mathbb{N} .
$$

Our main result, Theorem 1.3, follows directly from Lemma 2.1 and the following proposition.

Proposition 3.2. Suppose that (A1) through (A3) hold and $\mu_{0}$ is not concentrated at 0 and an element of $\mathcal{M}_{r}^{1}(\mathbb{R})$ for some $r \in(\vartheta, 2]$. Then $Z_{n}^{*} \stackrel{\mathbb{P}}{\rightarrow} Z$ as $n \rightarrow \infty$ for some non-degenerate random variable $Z$ and

$$
m(\vartheta)^{-\frac{n}{\vartheta}}(n \delta)^{\frac{3}{2 \vartheta}} \sum_{u \in \mathcal{I}_{n \delta}} e^{-S(u)} X_{u} \stackrel{d}{\rightarrow} Z,
$$

i.e., (2.1) holds.

The bulk of the proof of this proposition can be adopted from the proof of Theorem 2.5 in [17], however at some points changes are needed. In what follows, we repeat the major steps of the proof of the cited theorem adjusted to the situation here and point out the changes that are required.

Define the following point processes on $\mathbb{R}^{2}$ :

$$
\mathcal{Z}_{\infty}^{*}:=\sum_{k \in \mathbb{N}} \delta_{\left(P_{k}, X_{k}\right)} \quad \text { and } \quad \mathcal{Z}_{n}^{*}:=\sum_{|u|=n} \delta_{\left(V_{n}(u), X_{u}\right)}, \quad n \in \mathbb{N}_{0} .
$$


Lemma 3.3. Suppose that the assumptions of Proposition 3.2 are satisfied. Then $\int f(x, y) \mathcal{Z}_{n}^{*}(\mathrm{~d} x, \mathrm{~d} y) \rightarrow \int f(x) \mathcal{Z}_{\infty}^{*}(\mathrm{~d} x, \mathrm{~d} y)$ for all bounded continuous functions $f: \mathbb{R}^{2} \rightarrow$ $\mathbb{R}$ satisfying $f(x, y)=0$ for all sufficiently large $x$.

Source. The lemma is a special case of Lemma 5.2 in [17].

Lemma 3.4. Under the assumptions of Proposition 3.2, for any $\delta>0$ and any measurable $h: \mathbb{R} \mapsto \mathbb{R}$ satisfying $0 \leq h_{K} \leq \mathbb{1}_{[K, \infty)}$, we have

$$
\lim _{K \rightarrow \infty} \limsup _{n \rightarrow \infty} \mathbb{P}\left(\left|\sum_{|u|=n} e^{-\frac{1}{\vartheta} V_{n}(u)} h_{K}\left(V_{n}(u)\right) X_{u}\right|>\delta\right)=0 .
$$

Proof. The lemma follows from (the proof of) Lemma 5.3 in [17], except at one point in the proof where the Topchii-Vatutin inequality is used (Lemma A.1 in the cited reference). The use of the latter inequality has to be replaced by an application of (1.11) The rest of the proof carries over without changes.

We are now ready to prove Proposition 3.2.

Proof of Proposition 3.2. Recall that $0<\vartheta<r \leq 2$. Let $\beta_{0}:=\frac{r}{\vartheta}>1$. Given $\mathcal{Z}_{\infty}^{\circ}$, for each $n \in \mathbb{N}$, the random variable $Z_{n}^{*}$ is a sum of independent random variables (centered in the case $\vartheta \geq 1$ ). Then, for any $\delta>0$ and any $n, m \in \mathbb{N}$ with $m \leq n$, by (1.11),

$$
\mathbb{P}\left(\left|Z_{n}^{*}-Z_{m}^{*}\right|>\delta \mid \mathcal{Z}_{\infty}^{\circ}\right) \leq 2 \delta^{-r} \mathbb{E}\left[|X|^{r}\right] \cdot \sum_{k=m+1}^{n} e^{-\beta_{0} P_{k}}
$$

and the second term converges to zero as $m, n \rightarrow \infty$ by (3.6). Hence, conditionally given $\mathcal{Z}_{\infty}^{\circ},\left(Z_{n}^{*}\right)_{n \in \mathbb{N}_{0}}$ forms a Cauchy sequence in probability and thus converges in probability. We denote the limit in probability of the sequence $\left(Z_{n}^{*}\right)_{n \in \mathbb{N}_{0}}$ by $Z$. We now argue why $Z$ is not degenerate at a single point. Indeed, if $\mu_{0}$ is not concentrated in a single point, then given $\mathcal{Z}_{\infty}^{\circ}, Z$ is the limit in probability of a weighted sum of i.i.d. non-degenerate random variables and, hence, is non-degenerate. (To check this more formally, consider the Fourier transform of $Z_{n}^{*}$ conditionally given $\mathcal{Z}_{\infty}^{\circ}$ and notice that the limit of the Fourier transform cannot have absolute value 1 everywhere on $\mathbb{R}$ with probability one.) If, on the other hand, $\mu_{0}=\delta_{a} \in \mathcal{M}_{\gamma}^{1}(\mathbb{R})$ for some $a \in \mathbb{R}$, then, by assumption, $a \neq 0$. Since elements of $\mathcal{M}_{\gamma}^{1}(\mathbb{R})$ are centered if $\gamma>1$, this forces $\gamma \leq 1$. In particular, $\vartheta<\gamma \leq 1$. Then $Z$ equals $a$ times the a.s. convergent infinite series $\sum_{k \in \mathbb{N}} e^{-\beta P_{k}}$, see (3.6). This series is not a. s. constant as the points $P_{1}, P_{2}, \ldots$ are the points of a decorated Poisson point process [21, Theorem 1.1].

The proof of the second part is based on the decomposition

$$
\begin{aligned}
(m(\vartheta))^{-\frac{n}{\vartheta}} n^{\frac{3}{2 \vartheta}} \sum_{u \in \mathcal{I}_{n \delta}} e^{-S(u)} X_{u}= & \sum_{u \in \mathcal{I}_{n \delta}} e^{-\frac{1}{\vartheta} V_{n}(u)} X_{u} \\
= & \sum_{u \in \mathcal{I}_{n \delta}} e^{-\frac{1}{\vartheta} V_{n}(u)} f_{K}\left(V_{n}(u)\right) X_{u} \\
& +\sum_{u \in \mathcal{I}_{n \delta}} e^{-\frac{1}{\vartheta} V_{n}(u)}\left(1-f_{K}\left(V_{n}(u)\right)\right) X_{u} \\
= & : Z_{n, K}+R_{n, K},
\end{aligned}
$$

where $f_{K}$ is a continuous function such that $\mathbb{1}_{(-\infty, K]} \leq f_{K} \leq \mathbb{1}_{(-\infty, K+1]}$. The remainder of the proof is based on an application of Theorem 4.2 in [9]. In view of Lemma 3.4, the cited theorem gives the assertion once we have shown the following two assertions: 
1. $Z_{n, K} \stackrel{\text { d }}{\rightarrow} Z_{K}^{*}$ as $n \rightarrow \infty$ for every fixed $K>0$ where $Z_{K}^{*}$ is some finite random variable;

2. $Z_{K}^{*} \stackrel{P}{\rightarrow} Z$ as $K \rightarrow \infty$.

The first assertion is a consequence of Lemma 3.3. Indeed, the function on $\mathbb{R}^{2}$ that maps $(x, y)$ to $e^{-\frac{1}{9} x} f_{K}(x) y$ is continuous and vanishes for all sufficiently large $x$. Therefore, Lemma 3.3 yields

$$
\begin{aligned}
Z_{n, K} & =\sum_{u \in \mathcal{I}_{n \delta}} e^{-\frac{1}{\vartheta} V_{n}(u)} f_{K}\left(V_{n}(u)\right) X_{u} \\
& =\int e^{-\frac{1}{\vartheta} x} f_{K}(x) y \mathcal{Z}_{n}^{*}(\mathrm{~d} x, \mathrm{~d} y) \stackrel{\triangleleft}{\rightarrow} \int e^{-\frac{1}{\vartheta} x} f_{K}(x) y \mathcal{Z}_{\infty}^{*}(\mathrm{~d} x, \mathrm{~d} y)=: Z_{K}^{*} .
\end{aligned}
$$

The second assertion can be proved similarly as in the proof of Theorem 2.5 in [17]. More precisely, it follows from the dominated convergence theorem once we have proved that

$$
\mathbb{P}\left(\left|Z-Z_{K}^{*}\right|>\varepsilon \mid \mathcal{Z}_{\infty}^{\circ}\right) \rightarrow 0 \text { a.s. }
$$

as $K \rightarrow \infty$ for every $\varepsilon>0$. Now fix $\varepsilon>0$ and observe that

$$
\begin{aligned}
\mathbb{P}\left(\left|Z-Z_{K}^{*}\right|>\varepsilon \mid \mathcal{Z}_{\infty}^{\circ}\right) & \leq \mathbb{P}\left(\left\{\left|Z_{n}^{*}-Z_{K}^{*}\right|>\varepsilon \text { for infinitely many } n\right\} \mid \mathcal{Z}_{\infty}^{\circ}\right) \\
& =\mathbb{E}\left[\liminf _{n \rightarrow \infty} \mathbb{1}_{\left\{\left|Z_{n}^{*}-Z_{K}^{*}\right|>\varepsilon\right\}} \mid \mathcal{Z}_{\infty}^{\circ}\right] \\
& \leq \liminf _{n \rightarrow \infty} \mathbb{P}\left(\left|Z_{n}^{*}-Z_{K}^{*}\right|>\varepsilon \mid \mathcal{Z}_{\infty}^{\circ}\right) \\
& \leq \liminf _{n \rightarrow \infty} \varepsilon^{-r} \mathbb{E}\left[\left|Z_{n}^{*}-Z_{K}^{*}\right|^{r} \mid \mathcal{Z}_{\infty}^{\circ}\right],
\end{aligned}
$$

where Fatou's lemma gives the second inequality and Markov's inequality the third. Now given a realization $P_{1} \leq P_{2} \leq \ldots$ of the point process $\mathcal{Z}_{\infty}^{\circ}$, we can choose $n \in \mathbb{N}$ such that $P_{n}>K+1$. Then

$$
\begin{aligned}
\mathbb{E}\left[\left|Z_{n}^{*}-Z_{K}^{*}\right|^{r} \mid \mathcal{Z}_{\infty}^{\circ}\right] & =\mathbb{E}\left[\left|\sum_{k=1}^{n} e^{-\frac{P_{k}}{\vartheta}}\left(1-f_{K}\left(P_{k}\right)\right) X_{k}\right|^{r} \mid \mathcal{Z}_{\infty}^{\circ}\right] \\
& \leq 2 \sum_{k=1}^{n} e^{-\frac{r}{\vartheta} P_{k}}\left(1-f_{K}\left(P_{k}\right)\right)^{r} \mathbb{E}\left[\left|X_{k}\right|^{r}\right],
\end{aligned}
$$

where we have used inequality (1.11). The latter can be estimated as follows

$$
\sum_{k=1}^{n} e^{-\frac{p}{\vartheta} P_{k}}\left(1-f_{K}\left(P_{k}\right)\right)^{r} \mathbb{E}\left[\left|X_{k}\right|^{r}\right] \leq \mathbb{E}\left[\left|X_{1}\right|^{r}\right] \sum_{k \geq 1: P_{k}>K} e^{-\frac{r}{\vartheta} P_{k}} \rightarrow 0 \quad \text { a.s. }
$$

as $K \rightarrow \infty$ by (3.6) since $\frac{r}{\vartheta}>1$.

\section{References}

[1] Elie Aïdékon, Convergence in law of the minimum of a branching random walk, Ann. Probab. 41 (2013), no. 3A, 1362-1426. MR-3098680

[2] Gerold Alsmeyer and Matthias Meiners, Fixed points of the smoothing transform: two-sided solutions, Probab. Theory Related Fields 155 (2013), no. 1-2, 165-199. MR-3010396

[3] Gerold Alsmeyer and Uwe Rösler, The best constant in the Topchii-Vatutin inequality for martingales, Statist. Probab. Lett. 65 (2003), no. 3, 199-206. MR-2018031

[4] Federico Bassetti and Lucia Ladelli, Self-similar solutions in one-dimensional kinetic models: a probabilistic view, Ann. Appl. Probab. 22 (2012), no. 5, 1928-1961. MR-3025685

[5] Federico Bassetti, Lucia Ladelli, and Daniel Matthes, Central limit theorem for a class of onedimensional kinetic equations, Probab. Theory Related Fields 150 (2011), no. 1-2, 77-109. MR-2800905 
Self-similar solutions to kinetic-type evolution equations

[6] Federico Bassetti, Lucia Ladelli, and Daniel Matthes, Infinite energy solutions to inelastic homogeneous Boltzmann equations, Electron. J. Probab. 20 (2015), no. 89, 34. MR-3399825

[7] Federico Bassetti and Eleonora Perversi, Speed of convergence to equilibrium in Wasserstein metrics for Kac-like kinetic equations, Electron. J. Probab. 18 (2013), no. 6, 35. MR-3024100

[8] John D. Biggins and Andreas E. Kyprianou, Fixed points of the smoothing transform: the boundary case, Electron. J. Probab. 10 (2005), no. 17, 609-631 (electronic). MR-2147319

[9] Patrick Billingsley, Convergence of probability measures, John Wiley \& Sons, Inc., New York-London-Sydney, 1968. MR-0233396

[10] N. H. Bingham, C. M. Goldie, and J. L. Teugels, Regular variation, Encyclopedia of Mathematics and its Applications, vol. 27, Cambridge University Press, Cambridge, 1987. MR-1015093

[11] A. V. Bobylev, C. Cercignani, and I. M. Gamba, On the self-similar asymptotics for generalized nonlinear kinetic Maxwell models, Comm. Math. Phys. 291 (2009), no. 3, 599-644. MR2534787

[12] Kamil Bogus, Dariusz Buraczewski, and Alexander Marynych, Self-similar solutions of kinetictype equations: the boundary case, Stochastic Process. Appl. 130 (2020), no. 2, 677-693. MR-4046515

[13] Dariusz Buraczewski, Alexander Iksanov, and Bastien Mallein, On the derivative martingale in a branching random walk, arXiv:2002.05215, 2020.

[14] Yuan Shih Chow and Henry Teicher, Probability theory, third ed., Springer Texts in Statistics, Springer-Verlag, New York, 1997, Independence, interchangeability, martingales. MR1476912

[15] Bertram Düring, Daniel Matthes, and Giuseppe Toscani, Kinetic equations modelling wealth redistribution: a comparison of approaches, Phys. Rev. E (3) 78 (2008), no. 5, 056103, 12. MR-2551376

[16] Theodore E. Harris, The theory of branching processes, Die Grundlehren der Mathematischen Wissenschaften, Bd. 119, Springer-Verlag, Berlin; Prentice-Hall, Inc., Englewood Cliffs, N.J., 1963. MR-0163361

[17] Alexander Iksanov, Konrad Kolesko, and Matthias Meiners, Fluctuations of Biggins' martingales at complex parameters, Ann. Inst. Henri Poincaré Probab. Stat. 56 (2020), no. 4, 2445-2479. MR-4164844

[18] M. Kac, Foundations of kinetic theory, Proceedings of the Third Berkeley Symposium on Mathematical Statistics and Probability, 1954-1955, vol. III, University of California Press, Berkeley and Los Angeles, 1956, pp. 171-197. MR-0084985

[19] Tatsuo Kawata, Fourier analysis in probability theory, Academic Press, New York-London, 1972, Probability and Mathematical Statistics, No. 15. MR-0464353

[20] J. F. C. Kingman, Ergodic properties of continuous-time Markov processes and their discrete skeletons, Proc. London Math. Soc. (3) 13 (1963), 593-604. MR-0154334

[21] Thomas Madaule, Convergence in law for the branching random walk seen from its tip, J. Theoret. Probab. 30 (2017), no. 1, 27-63. MR-3615081

[22] Daniel Matthes and Giuseppe Toscani, On steady distributions of kinetic models of conservative economies, J. Stat. Phys. 130 (2008), no. 6, 1087-1117. MR-2379241

[23] Lorenzo Pareschi and Giuseppe Toscani, Self-similarity and power-like tails in nonconservative kinetic models, J. Stat. Phys. 124 (2006), no. 2-4, 747-779. MR-2264624

[24] H.-J. Schuh, Sums of i.i.d. random variables and an application to the explosion criterion for Markov branching processes, J. Appl. Probab. 19 (1982), no. 1, 29-38. MR-0644417

Acknowledgments. The authors thank an anonymous referee for a very thorough report including suggestions that helped simplifying the presentation. D. B. and K. K. were partially supported by the National Science Center, Poland (Sonata Bis, grant number DEC-2014/14/E/ST1/00588). The research of M. M. was supported by DFG Grant ME 3625/3-1. The work was initiated while D. B. was visiting Innsbruck in May 2019. He gratefully acknowledges hospitality and the financial support again by DFG Grant ME 3625/3-1. 